\title{
Hans Bethe on solar neutrinos
}

\section{Whatever can account for the much smaller flux of neutrinos from the Sun than the theorists predict?} Now, at last, there may be an answer.

NoBODy forgets that the doctrine that the Sun's energy is the product of thermonuclear fusion within the core of the star was the product, more than half a century ago, of the ingenious mind of the young emigrant physicist Hans Bethe, then newly a refugee from Hitler's Germany.

Bethe's theory has been, of course, a huge success; not merely does it successfully account for astrophysical phenomena other than the production of energy by stars, but it accounts splendidly for the properties of the Sun, the most closely observed of all stars, except in one respect: the output of neutrinos from the Sun, as measured by the experiments due to $\mathrm{R}$. Davis $\mathrm{Jr}$ in which the conversion of chlorine-37 into argon-37 under the influence of the solar flux of neutrinos is found to be less than a third of what Bethe's followers would predict.

It would be a delightful happening if the puzzle of the missing neutrinos from the Sun proves to be resolved by Bethe's most recent scientific paper, an elegant piece of argument in Physical Review Letters for 24 March $(56,1305 ; 1986)$. Not often can a person hope to provide care and maintenance for a theory after the lapse of so much time.

Bethe's starting point is twofold, the discrepancy between the measured and expected flux of neutrinos from the Sun and what he calls "a very important paper" by two Soviet physicists, S.P. Mikheyev and A. Yu. Smirnov, said to have been presented at a conference at Helsinki in June last year. Bethe says that he has come to the same conclusion by different arguments, which is generous of him considering that, on the way, he corrects the sign of a crucial term in an equation which is essential to the physics of his result.

Several concepts enter, of which the simplest is that the conversion of chlorine37 into argon-37 (which is detectable atom by atom because argon- 37 is both gaseous and radioactive) is a means of recognizing electron neutrinos, the partner particles of ordinary electrons. But the partners of muons are different, and called muon neutrinos. Indeed, the interaction of a neutrino with a nucleus of chlorine- 37 is the inverse of the radioactive decay of argon-37, which explains why muon neutrinos are not detected in Davis's tank full of chlorinated hydrocarbon.

The second concept, less familiar, is that of neutrino mixing, predictable since the time when people became familiar with Dirac's theory of the relativistic fermion, taken as a credible fact of life since the early $1960 \mathrm{~s}$. The idea is that because neutrinos (unlike electrons) have no electric charge, those actually observed in laboratory experiments will be superpositions (in the quantum sense) of whatever neutrino states there may be. (Since there is a third generation of charged fermions, called tauons, there is also a third generation of neutrinos that may participate in the mixing.)

Hitherto, neutrino mixing has been one way of accounting for the missing neutrinos from the Sun. People have supposed that the neutrinos reaching the Earth are superpositions of electron and muon neutrinos, have reckoned that only the electron component of the compound state will be detectable and have gone on to guess at what admixture of the muon-neutrino state will account for the discrepancy; the snag has been that this estimate conflicts with that obtained from measurements near nuclear reactors (where it should be possible directly to measure the way in which the ratio changes with distance from the reactor, "oscillating" in the process).

What Bethe now does is to calculate the behaviour of neutrinos in the deep interior of the Sun, the novel step in his calculation and that of his Soviet precursors. On the journey from the Sun to the Earth, where there is not much of any kind of matter, the properties of the neutrinos are not much affected by external events. But in the interior of the Sun, where the density is high, there is matter enough with which the neutrinos may interact. But muons are scarce, so that only the electron neutrino part of the mixed state of the neutrino will interact. It is as if, says Bethe, electron neutrinos experience a potential energy dependent on the density (or the electron density) of the matter in their locality. But this feeds back to affect the mixing ratio, with the result that observable neutrinos are more like muon neutrinos in the interior of the Sun (where the density of matter is greater and so the potential energy of the electron neutrinos that much greater).

Another way of putting this is that the effective mass of the electron neutrinos (for energy is mass) is greater in the interior of the Sun than in empty space. But do neutrinos have mass? Maybe a little, perhaps just enough to close the Universe. And if they do have mass, the masses of the electron and the muon neutrinos will be different while the masses of the measurable mixed states will be individually constant wherever they are measured, which leads quickly to the conclusion that in a region where the density of matter is great enough, electron neutrinos will be heavier than muon neutrinos if the opposite is true elsewhere. And that implies that within some critical radius within the Sun, electron neutrinos will be converted into muon neutrinos because they are more energetic, even though in empty space the energy balance goes the other way. In other words, there is a possibility that the electron neutrinos produced in the Sun's interior (predominantly by the conversion of boron- 8 into beryllium-8) will be converted into muon neutrinos so effectively that they will hardly have a chance to reach the Earth.

With this conclusion, Bethe nicely turns the problem on its head, supposing that the conversion of electron into muon neutrinos in the interior of the Sun accounts for Davis's discrepancy, and going on to ask what that implies for the properties of neutrinos. The result is startling, if only because it shows how much an ingenious person can extract from a working hypothesis as simple even as this. "Now I propose to take this theory seriously" is how Bethe puts it.

One conclusion (which hangs on the assumption that neutrinos have some mass, however small) is that electron neutrinos whose energy is small enough will emerge as such from the interior of the Sun; putting in numbers, only one in eight of the neutrinos from the conversion of boron- 8 to beryllium- 8 will reach the Earth. This is more than enough to explain Davis's discrepancy, but also suggests that the gallium experiment planned by J.H. Bahcall should be less affected.

But the conclusion that stands out is Bethe's estimate of the neutrino mass. If the tauon neutrino is ignored, and the less massive of the observable neutrinos assigned zero mass, the heavier observable neutrino (in which the muon neutrino predominates) has a mass of only $0.008 \mathrm{eV}$. This is much less than the mass required to close the Universe. It is also less than that measured in the few experiments reporting a positive result. Bethe may well be right in claiming that astrophysics is a better way to a determination of the neutrino mass than any other kind of experiment.
John Maddox 